\title{
Why Targeting PSMA Is a Game Changer in the Management of Prostate Cancer
}

\author{
Nicholas M. Donin and Robert E. Reiter \\ Department of Urology, David Geffen School of Medicine at UCLA, Los Angeles, California
}

\begin{abstract}
Prostate-specific membrane antigen (PSMA) is a transmembrane glycoprotein that is highly expressed on prostate adenocarcinomas, exhibits only limited expression in benign and extraprostatic tissues, and thus represents an ideal target for the diagnosis and management of prostate cancer. Since its discovery over $30 \mathrm{y}$ ago, significant effort has been made to develop clinical technology targeting PSMA. The last 5 y have seen an explosion of development of new agents targeting PSMA for diagnostic and therapeutic use. Imaging agents targeting PSMA have been developed for SPECT and PET platforms. PSMA PET imaging appears to outperform traditional imaging in the high-risk localized-disease state, in patients with biochemical recurrence after treatment, and in advanced disease. To date, most of the reported clinical studies of therapeutic agents have used PSMA-targeted radiometals to deliver $\beta$-radiation to metastatic disease sites, with ${ }^{177} \mathrm{Lu}$ being the most widely investigated therapeutic radioisotope. Studies of both antibodies and small-molecule agents have been published and have demonstrated encouraging results. Safety appears generally limited to mild transient bone marrow toxicity and xerostomia because of uptake of the small-molecule agents in the salivary glands. Radiologic responses can be dramatic, and decreases in pain have been observed. The effect on overall survival, however, has yet to be demonstrated.
\end{abstract}

Key Words: PSMA; prostate cancer; urology

J Nucl Med 2018; 59:177-182

DOI: 10.2967/jnumed.117.191874

A

dvances in the early detection and treatment of prostate cancer have resulted in a $50 \%$ decrease in mortality from prostate cancer in the United States over the last 25 y (1). Despite these strides, a subset of men either present with de novo metastatic disease or will progress to the metastatic disease state despite attempts to cure at the localized-disease state. Although androgen deprivation therapy slows disease progression, metastatic tumors ultimately develop castration resistance and are generally incurable. The past decade has seen the development of treatments of various modalities for metastatic castration-resistant prostate cancer (mCRPC), including second-generation endocrine manipulation, cytotoxic chemotherapy, cellular immunotherapy, and ${ }^{223}$ Ra-dichloride. Although these agents have been shown to prolong overall survival,

Received Jul. 6, 2017; revision accepted Sep. 13, 2017.

For correspondence or reprints contact: Nicholas M. Donin, David Geffen School of Medicine at UCLA, 44151 15th St. W., Lancaster, CA 93534.

E-mail: nicholasdonin@gmail.com

Published online Oct. 6, 2017.

COPYRIGHT (c) 2018 by the Society of Nuclear Medicine and Molecular Imaging. the benefits conferred are modest, and mCRPC remains a leading cause of cancer death, killing over 300,000 men worldwide annually (2).

\section{BACKGROUND}

Prostate-specific membrane antigen (PSMA) is a 750-amino-acid type II transmembrane glycoprotein encoded by the folate hydrolase 1 gene located on the short arm of chromosome 11 (3). The name PSMA is a misnomer, as the protein is expressed not only on both benign and malignant prostate epithelium but also on a variety of extraprostatic tissues, including the proximal renal tubules (4), jejunal brush border (5), salivary glands (5), and neovasculature of several solid tumors (6). Structurally, the transmembrane protein consists of a 19-amino-acid intracellular domain, a 24-amino-acid transmembrane domain, and a large, 707-amino-acid, extracellular domain (7).

Histologically, PSMA is detectable at modest levels in the epithelium of benign prostate tissue but demonstrates 100- to 1,000-fold expression on the epithelium of prostate adenocarcinomas $(4,8)$. It is expressed in most tumors, and a positive correlation has been observed between higher PSMA expression and various measures of tumor aggressiveness, including Gleason grade (8), tumor stage (9), biochemical recurrence (10), and castration resistance (11). The cytoplasmic domain of PSMA contains a motif that results in internalization of bound PSMA via clathrincoated pits (12). This process provides the possibility that PSMAtargeting agents might be internalized and concentrated within tumor cells. PSMA is thus an attractive target for diagnostic and therapeutic targeting for several reasons, including high expression on prostate cancer cells, limited expression on benign prostate tissue, limited expression on nonprostate tissue, an extracellular domain that can be targeted by antibodies, a well-characterized binding site that can be targeted by small-molecule ligands, and a motif that results in internalization of bound agents and concentration within malignant cells.

\section{TARGETING AGENTS}

\section{Antibodies}

The first steps in targeting PSMA took place in the late 1980s and involved generation of the 7E11-C35 antibody, which is specific to an epitope at the intracellular domain of PSMA (13). This antibody was then labeled with ${ }^{111} \mathrm{In}$, allowing for use with SPECT imaging. This agent, known as capromab pendetide (ProstaScint; Aytu BioScience, Inc.) was Food and Drug Administrationapproved in 1996 for the detection of soft-tissue metastases. When ProstaScint was evaluated for use in initial staging, 2 large multicenter trials demonstrated sensitivities of $52 \%-62 \%$ and specificities of $72 \%-96 \%$ using pelvic lymphadenectomy as the truth standard, 
outperforming both CT and MRI (14). When used for the detection of suspected recurrent or residual cancer after treatment of the primary tumor, ProstaScint demonstrated a sensitivity of $49 \%$ $77 \%$ and specificity of $35 \%-71 \%$ (14). Despite this modestly improved performance compared with traditional imaging, the relatively poor sensitivity of ProstaScint in the setting of low prostate-specific antigen (PSA) levels, difficulties in anatomic localization because of the limitations of SPECT, and significant operator dependence resulted in relatively limited use.

To improve on these limitations, huJ591, a humanized monoclonal IgG1 antibody that binds an extracellular epitope of PSMA, has been developed for use with both $\gamma$ - and photon-emitting metalloradionuclide agents (15). The antibody has been used in several early-phase clinical trials for both imaging and therapy (Supplemental Table 1; supplemental materials are available at http://jnm.snmjournals.org) (16-19). Because IgG antibodies are not filtered at the glomerulus and remain within the blood pool for several days, imaging must be performed 6-8 d after infusion to allow clearance of the antibody from the blood pool. An 80$\mathrm{kDa}$ minibody that has been genetically engineered to lack the Fc-receptor domain, known as ${ }^{89} \mathrm{Zr}$-Df-IAB2M, was synthesized with the aim of generating faster blood clearance in order to allow for imaging at a shorter time interval (20). The initial studies suggest that a 48-h wait time between infusion and imaging may still be required.

\section{Small-Molecule PSMA Ligands}

Characterization of the active substrate-recognition site of the PSMA molecule has allowed for the development of numerous agents engineered to bind to this site. Small molecules have the theoretic advantage over antibodies of achieving better tumor penetration and faster clearance from the blood pool, allowing for infusion and imaging to be performed during a single patient-visit. Numerous small-molecule agents, labeled with a range of radionuclides for use with both SPECT imaging and PET imaging, as well as for the delivery of radiometallonuclides for treatment purposes, have been developed and are in various stages of clinical use (Table 1 and Supplemental Table 1). Several of the more promising small-molecule agents are discussed below.

${ }^{68} \mathrm{Ga}$-PSMA-HBED-CC $\left({ }^{68} \mathrm{Ga}-\mathrm{PSMA}-11\right)$ is the most widely used PET agent for PSMA-targeted imaging. First described in 2012, the agent consists of the HBED-CC chelator to which the ${ }^{68} \mathrm{Ga}$ is bound, a lipophilic linker, and a urea-based Glu-CO-NHLys motif that binds to the active site of the PSMA molecule (21). It displays low-level natural uptake in the kidneys, salivary glands, lacrimal glands, liver, spleen, and bowel and has demonstrated the ability to detect prostate cancer within the prostate gland, within

\section{NOTEWORTHY}

- PSMA has been a molecular target of interest in prostate cancer since its discovery in 1986.

- A variety of antibodies and small-molecule imaging agents targeting PSMA have demonstrated excellent early results for a variety of disease states.

- Radioimmunotherapeutic and radioligand agents are currently being investigated for use in the mCRPC setting, with several demonstrating encouraging clinical responses.

- The effect of these agents on overall survival remains a subject of investigation. small nodal metastases, within bony lesions, and even within more widespread dedifferentiated tumors (22-24). Because the agent is filtered at the glomerulus, high levels of activity are present in the urine, potentially making the detection of local recurrences more difficult; however, nodal metastases near the bladder have been detected (24). A systematic review was published in 2016 by Perera et al. evaluating the sensitivity and specificity of ${ }^{68} \mathrm{G}-$ PSMA-HBED-CC in various clinical settings (25). Further details of the agent's performance will be discussed below.

Another area of robust investigation in PSMA ligands involves agents that use ${ }^{18} \mathrm{~F}$, a radionuclide that has several theoretic advantages over ${ }^{68} \mathrm{Ga}$, including better image resolution because of a shorter positron range and a higher positron yield. Like the ${ }^{68} \mathrm{Ga}$ agents, ${ }^{18} \mathrm{~F}$ agents can be infused and imaged at the same visit. The first of these agents to be tested clinically was ${ }^{18} \mathrm{~F}$-DCFBC, which has been evaluated both for disease assessment within the gland (26) and for detection of metastases $(27,28)$. Although initial results were encouraging, the significant blood-pool activity of the agent prompted efforts to refine it and improve its performance. The result of these efforts was a second-generation agent, 2-(3(1-carboxy-5-[(6- ${ }^{18} \mathrm{~F}$-fluoro-pyridine-3-carbonyl)-amino]-pentyl)ureido)-pentanedioic acid $\left({ }^{18} \mathrm{~F}-\mathrm{DCFPyL}\right)$, which was initially evaluated in a cohort of 9 patients with metastatic disease $(29,30)$. As hoped, the agent demonstrated a marked improvement in maximum tumorto-blood-pool uptake ratios (30), allowing for improved visual conspicuity of suspected disease. Another agent, ${ }^{18} \mathrm{~F}$-PSMA-1007, is also in development and has shown the ability to detect micrometastases (31) in the biochemical recurrence setting.

\section{DIAGNOSTIC TARGETING}

\section{High-Risk Initial Diagnosis}

At least 4 published studies have evaluated the performance of PSMA-targeted agents for use in initial staging of intermediateand high-risk disease for which histopathologic correlation was performed (32-35). These studies used the ${ }^{68}$ Ga-PSMA-HBED$\mathrm{CC}$ tracer, and in all 4 studies, PET with this tracer outperformed traditional CT or MRI for lymph node staging, with both improved sensitivity and improved specificity on both a per-patient and a per-template basis. On a per-patient basis, sensitivities ranged from $33 \%$ to $91 \%$ and specificities from $67 \%$ to $100 \%$. On a per-template basis, sensitivities ranged from $74 \%$ to $86 \%$ and specificities from $88 \%$ to $99 \%$.

\section{Biochemical Recurrence}

At present, biochemical recurrence can be detected long before imaging technology allows anatomic localization of disease. This provides challenges for management because local radiotherapy, which is known to prolong both disease-free and overall survival (36), is most effective when applied at low PSA levels, although local recurrence presently can only be inferred from pathologic data and PSA kinetics. Given reticence to proceed with local salvage radiotherapy without definitive evidence of local recurrence, imaging technology to improve the localization of disease recurrence is of paramount interest and represents one of the most robust areas of PSMA-targeted imaging research. Afshar-Oromieh et al. published the largest study for this indication, an analysis of 1,007 men with biochemically recurrent disease (22) who underwent PET using the ${ }^{68} \mathrm{G}-\mathrm{PSMA}-\mathrm{HBED}-\mathrm{CC}$ tracer. In $79.5 \%$ of patients, at least 1 lesion suggestive of prostate cancer was identified, including lesions in bone, soft tissue, and viscera. There was 


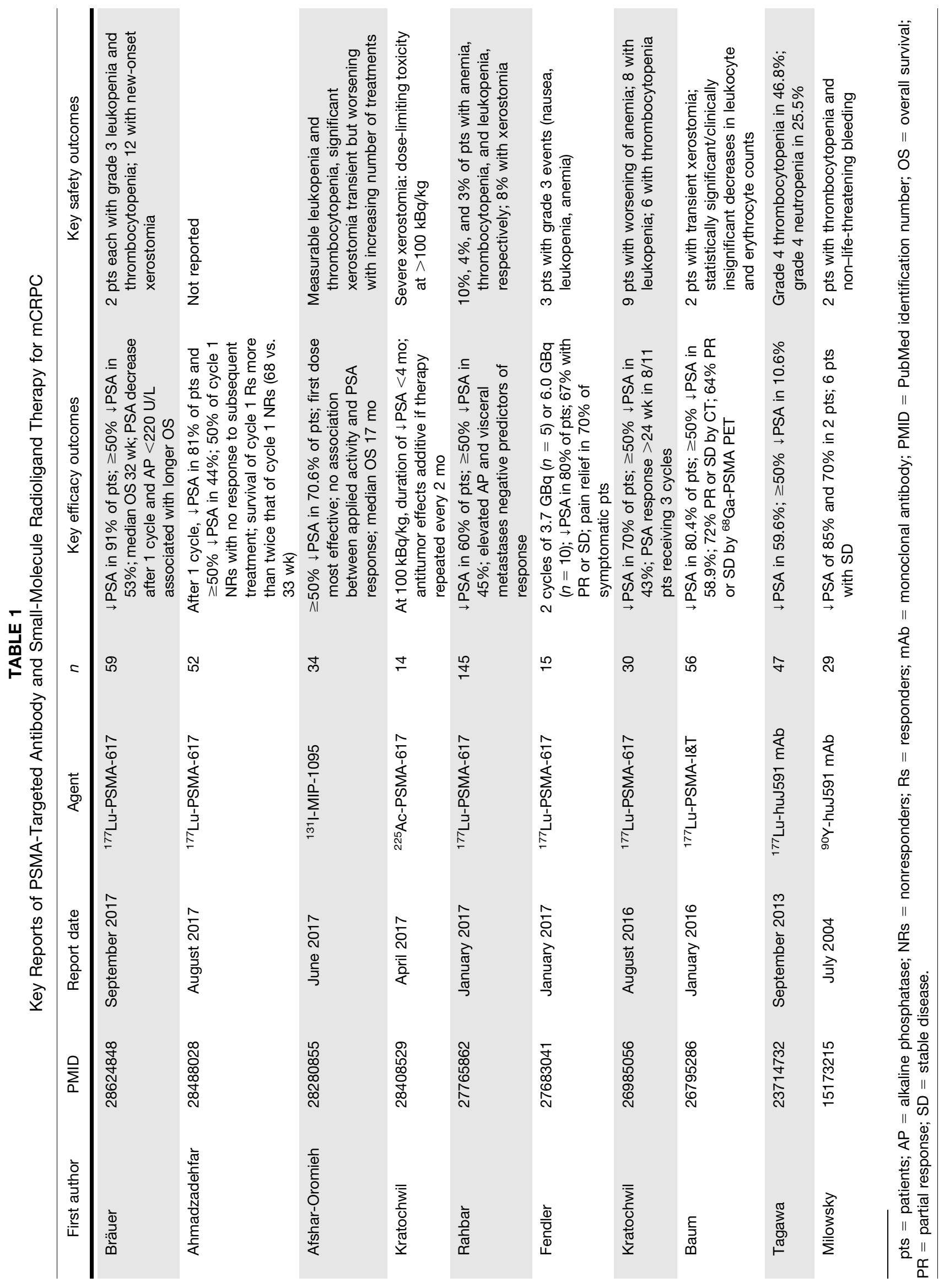


a clear relationship between the likelihood of a positive scan result and PSA level: a $46 \%$ likelihood at a level of $0.5 \mathrm{ng} / \mathrm{mL}$ or less, $73 \%$ at $0.51-1.0 \mathrm{ng} / \mathrm{mL}, 80 \%$ at $1.1-2.0 \mathrm{ng} / \mathrm{mL}, 86 \%$ at $2.1-3.0$ $\mathrm{ng} / \mathrm{mL}, 91 \%$ at $3.1-5.0 \mathrm{ng} / \mathrm{mL}, 94 \%$ at $5.1-7.0 \mathrm{ng} / \mathrm{mL}, 91 \%$ at 7.1-10 ng/mL, and $96 \%$ at more than $10 \mathrm{ng} / \mathrm{mL}$. A multivariable logistic regression analysis found that log PSA and receipt of androgen deprivation therapy predicted a positive scan result but that Gleason score did not. These results are generally consistent with a report from Eiber et al., who published on a cohort of 248 consecutive patients with biochemical recurrence after radical prostatectomy. PSMA PET is especially relevant at low PSA values given that guidelines for salvage radiotherapy recommend treatment at a PSA level of less than 0.5 and that other PET tracers, such as ${ }^{18} \mathrm{~F}$-choline, demonstrated limited sensitivity at this level (19\%-36\%). Apropos to this point, Bluemel et al. published a report on 125 patients with biochemical recurrence after radiation or radical prostatectomy who underwent ${ }^{18} \mathrm{~F}$-choline PET and, if negative, ${ }^{68} \mathrm{G}-\mathrm{PSMA}$-imaging and therapy (I\&T) PET/CT ( ${ }^{177}$ Lu-DOTAGA) (37). These investigators found that ${ }^{68} \mathrm{G}$-PSMA-I\&T detected sites of BCR in $44 \%$ of patients with a negative ${ }^{18} \mathrm{~F}$-choline PET/CT result (37), with the incremental benefit of the PSMA study being most pronounced in the subset of patients with a PSA level of less than $1 \mathrm{ng} / \mathrm{mL}$.

\section{Metastatic}

Most published studies have demonstrated that PSMA-targeting agents, both antibody-based and small-molecule ligands, are safe and provide high sensitivity and specificity for staging lymph node, soft-tissue, and bony metastases $(20,27,29,30,38-41)$. One study, by Rowe et al.- a head-to-head comparison of the performance of conventional imaging versus ${ }^{18} \mathrm{~F}$-DCFPyL-demonstrated some of the key considerations in this area (30). First, ${ }^{18} \mathrm{~F}$ DCFPyL detected over 3 times the number of metastatic lesions detected by conventional imaging. Second, the authors discussed the ability of ${ }^{18} \mathrm{~F}-\mathrm{DCFPyL}$ to detect metastases in small lymph nodes, noting the failure of simple size cutoff to distinguish between benign and malignant nodes. Finally, ${ }^{18} \mathrm{~F}-\mathrm{DCFPyL}$ detected metastatic disease in the periprostatic soft tissues, an area difficult to assess with either CT or MRI, and the authors highlighted a case in which a patient with normal results on pelvic MRI showed a perirectal metastasis on ${ }^{18} \mathrm{~F}$-DCFPyL PET. The main limitation of this study and others in this area is the lack of a systematic formal histologic evaluation on a lesion-by-lesion basis to serve as the truth standard. As such, the true performance of PSMA-targeted imaging for metastatic disease remains incompletely evaluated. Nevertheless, the initial studies provide strong preliminary evidence that PSMA-targeted imaging agents are likely to outperform traditional imaging procedures for the detection of metastatic disease. The value of molecular imaging to monitor therapy is, at present, unproven with respect to overall survival. Unfortunately, molecular imaging is not included in ongoing large trials on advanced disease.

\section{THERAPEUTIC TARGETING}

Targeted cancer therapy aims to achieve sensitive and specific on-target, on-tumor cell death while sparing normal tissues. Great effort has been made to develop agents that target PSMA for treatment in the mCRPC disease state, with the appreciation that prostate cancer is radiosensitive, prompting investigation into the use of radiopharmaceuticals as potential candidate effector agents.
Most published studies on nascent radiopharmaceuticals describe small-molecule agents that use ${ }^{177} \mathrm{Lu}$ as the radiometal, but earlyphase studies have evaluated antibody-based therapies as well. A summary of the key published reports is provided in Table 1. Several notable agents are discussed below. No randomized studies exist to date.

The first PSMA-targeted radioimmunotherapeutic studies used the huJ591 antibody. Two phase 1 dose-finding studies, one using ${ }^{90} \mathrm{Y}$ and one using ${ }^{177} \mathrm{Lu}$, were published $(18,19)$, followed by a phase 2 study using ${ }^{177} \mathrm{Lu}$ and published in 2013 by Tagawa et al. (17). In the latter, 47 patients were treated at 2 doses $(2,405$ and $2,590 \mathrm{MBq} / \mathrm{m}^{2}$ ). Key outcomes included a PSA decline of at least $50 \%$ in $10.6 \%$ of patients and a PSA decline of any amount in $59.6 \%$ of patients. Median overall survival for the entire cohort was $17.6 \mathrm{mo}$, with higher-dose patients surviving almost twice as long (21.8 vs. 11.9 mo). Myelosuppression was the main observed side effect, including grade 4 thrombocytopenia in almost half the patients, but was reversible. No significant hemorrhages occurred.

More recent attention has been focused on small-molecule PSMA-targeting radioligand therapies, many of which use theranostic agents. Theranostic agents are those in which the chelator is capable of binding radiometals for both imaging $\left({ }^{68} \mathrm{Ga}\right)$ and treatment $\left({ }^{177} \mathrm{Lu}\right)$. Like imaging agents, small-molecule radioligand agents have the advantage of clearing from the blood more quickly than antibodies, resulting in lower doses of radiation delivered to normal tissues.

The best-studied PSMA-targeted radioligand therapeutic agent is ${ }^{177} \mathrm{Lu}$-PSMA-617. The first reported cohort was published in 2015 (42), and since that time multiple investigator groups have published results evaluating this agent (43-46). The largest study to date is a retrospective multicenter cohort of 145 patients from 12 centers across Germany (47). Some variation in efficacy outcomes is seen across the studies: after a single treatment, 59\%$79 \%$ of patients experienced a PSA decrease, with $32 \%-45 \%$ of patients experiencing a decrease of at least $50 \%$. The studies by Rahbar et al. (47) and Kratochwil et al. (43) suggested that patients who receive multiple treatments continue to respond to subsequent treatments at a similar, if not increasing, rate. The large German multicenter study demonstrated that the presence of visceral metastases and an alkaline phosphatase level of at least $220 \mathrm{U} / \mathrm{L}$ predicted a lower rate of treatment response. The study by Ahmadzadehfar et al. found that responders to the initial cycle of treatment survived over twice as long as nonresponders (45). In all cohorts, leukopenia and thrombocytopenia were reported but were mild. Xerostomia was seen but was mild and transient and rarely required salivary replacement.

${ }^{177} \mathrm{Lu}-\mathrm{PSMA}-\mathrm{I} \& \mathrm{~T}$ is yet another PSMA-targeted radioligand therapeutic agent with early promising results. Baum et al. reported on a group of 56 patients with mCRPC who underwent multiple treatments (48). Overall, $80 \%$ of patients had a PSA decrease, with $59 \%$ having a decrease of more than $50 \%$. Again, mild, self-limited xerostomia was noted in 2 patients, with clinically insignificant decreases in leukocyte and erythrocyte counts. RECIST morphologic response assessment by $\mathrm{CT}$ demonstrated a partial response in $20 \%$, stable disease in $53 \%$, and progressive disease in $28 \%$, whereas response assessment by ${ }^{68}$ G-PSMA PET demonstrated a partial response in $56 \%$, stable disease in $8 \%$, and progressive disease in $36 \%$. The authors pointed out that changes detectable by SUVs on PET/CT may occur before changes in lesion or lymph node size and might be responsible for the discrepancy in response rates. 
The PSMA-617 agent has also been tested in early clinical trials with the $\alpha$-emitting radiometal ${ }^{225} \mathrm{Ac}$ in an attempt to reduce potential hematologic and salivary toxicities (because of the shorter range of $\alpha$-particles) and potentially to break through radioresistance to ${ }^{225} \mathrm{Lu}$. In a 14-patient dose-finding cohort, Kratochwil et al. determined that a dose of $100 \mathrm{kBq} / \mathrm{kg}$ was the maximum tolerable and that a schedule of dosing every 2 mo appeared feasible (49). Efficacy was suggested, and plans for further study are in progress.

Although dramatic radiologic responses have been noted in many of these early-phase PSMA-targeting radioligand and radioimmunotherapeutic trials, and results suggest a role for these agents in the management of mCRPC, there is, at present, no level 1 evidence demonstrating a benefit to overall survival. The impact of cotargeting approaches that create synthetic lethality from DNA damage with poly(ADP-ribose) polymerase inhibitors, next-generation androgen ablation, and platinum-based chemotherapies has not yet been explored.

\section{CONCLUSION}

PSMA is a promising molecular target in prostate cancer management for several reasons, including high levels of expression on most prostate cancer cells with limited expression on benign tissues, proven in vivo safety and feasibility in targeting PSMA using antibodies and small molecules, and a motif that provides for internalization and concentration of agents. Several studies have shown that the PET-based imaging assays outperform standard imaging techniques and that these assays appear poised to become a new standard in prostate cancer imaging. Early-phase therapeutic trials of unsealed radiometals have produced promising results in mCRPC; however, more study will be required to prove their effect on meaningful endpoints. PSMA targeting is likely to play a central role in prostate cancer management in the future.

\section{DISCLOSURE}

No potential conflict of interest relevant to this article was reported.

\section{REFERENCES}

1. Cancer facts \& figures 2017. American Cancer Society website. https://www.cancer. org/research/cancer-facts-statistics/all-cancer-facts-figures/cancer-facts-figures-2017. html. Accessed November 9, 2017.

2. Global cancer facts \& figures. American Cancer Society website. https://www. cancer.org/research/cancer-facts-statistics/global.html. Accessed November 9, 2017.

3. Rinker-Schaeffer CW, Hawkins AL, Su SL, et al. Localization and physical mapping of the prostate-specific membrane antigen (PSM) gene to human chromosome 11. Genomics. 1995;30:105-108.

4. Silver DA, Pellicer I, Fair WR, Heston WD, Cordon-Cardo C. Prostate-specific membrane antigen expression in normal and malignant human tissues. Clin Cancer Res. 1997;3:81-85.

5. Troyer JK, Beckett ML, Wright GL. Detection and characterization of the prostate-specific membrane antigen (PSMA) in tissue extracts and body fluids. Int J Cancer. 1995;62:552-558.

6. Baccala A, Sercia L, Li J, Heston W, Zhou M. Expression of prostate-specific membrane antigen in tumor-associated neovasculature of renal neoplasms. Urology. 2007;70:385-390.

7. Davis MI, Bennett MJ, Thomas LM, Bjorkman PJ. Crystal structure of prostatespecific membrane antigen, a tumor marker and peptidase. Proc Natl Acad Sci USA. 2005;102:5981-5986.

8. Bostwick DG, Pacelli A, Blute M, Roche P, Murphy GP. Prostate specific membrane antigen expression in prostatic intraepithelial neoplasia and adenocarcinoma. Cancer. 1998;82:2256-2261.
9. Perner S, Hofer MD, Kim R, et al. Prostate-specific membrane antigen expression as a predictor of prostate cancer progression. Hum Pathol. 2007;38:696-701.

10. Ross JS, Sheehan CE, Fisher HAG, et al. Correlation of primary tumor prostatespecific membrane antigen expression with disease recurrence in prostate cancer. Clin Cancer Res. 2003;9:6357-6362.

11. Wright GL, Grob BM, Haley C, et al. Upregulation of prostate-specific membrane antigen after androgen-deprivation therapy. Urology. 1996;48:326-334.

12. Rajasekaran SA, Anilkumar G, Oshima E, et al. A novel cytoplasmic tail MXXXL motif mediates the internalization of prostate-specific membrane antigen. Mol Biol Cell. 2003;14:4835-4845.

13. Horoszewicz JS, Kawinski E, Murphy GP. Monoclonal antibodies to a new antigenic marker in epithelial prostatic cells and serum of prostatic cancer patients. Anticancer Res. 1987;7:927-935.

14. Lamb HM, Faulds D. Capromab pendetide: a review of its use as an imaging agent in prostate cancer. Drugs Aging. 1998;12:293-304.

15. Smith-Jones PM, Vallabahajosula S, Goldsmith SJ, et al. In vitro characterization of radiolabeled monoclonal antibodies specific for the extracellular domain of prostate-specific membrane antigen. Cancer Res. 2000;60:5237-5243.

16. Osborne JR, Green DA, Spratt DE, et al. A prospective pilot study of ${ }^{89} \mathrm{Zr}-\mathrm{J} 591 /$ prostate specific membrane antigen positron emission tomography in men with localized prostate cancer undergoing radical prostatectomy. J Urol. 2014;191:1439-1445.

17. Tagawa ST, Milowsky MI, Morris M, et al. Phase II study of lutetium-177-labeled anti-prostate-specific membrane antigen monoclonal antibody J591 for metastatic castration-resistant prostate cancer. Clin Cancer Res. 2013;19:5182-5191.

18. Bander NH, Milowsky MI, Nanus DM, Kostakoglu L, Vallabhajosula S, Goldsmith SJ. Phase I trial of ${ }^{177}$ lutetium-labeled J591, a monoclonal antibody to prostate-specific membrane antigen, in patients with androgen-independent prostate cancer. J Clin Oncol. 2005;23:4591-4601.

19. Milowsky MI, Nanus DM, Kostakoglu L, Vallabhajosula S, Goldsmith SJ, Bander NH. Phase I trial of yttrium-90-labeled anti-prostate-specific membrane antigen monoclonal antibody J591 for androgen-independent prostate cancer. J Clin Oncol. 2004;22:2522-2531.

20. Pandit-Taskar N, O'Donoghue JA, Ruan S, et al. First-in-human imaging with ${ }^{89} \mathrm{Zr}$-Df-IAB2M anti-PSMA minibody in patients with metastatic prostate cancer: pharmacokinetics, biodistribution, dosimetry, and lesion uptake. J Nucl Med. 2016;57:1858-1864.

21. Eder M, Schäfer M, Bauder-Wüst U, et al. ${ }^{68} \mathrm{Ga}$-complex lipophilicity and the targeting property of a urea-based PSMA inhibitor for PET imaging. Bioconjug Chem. 2012;23:688-697.

22. Afshar-Oromieh A, Holland-Letz T, Giesel FL, et al. Diagnostic performance of ${ }^{68} \mathrm{Ga}$-PSMA-11 (HBED-CC) PET/CT in patients with recurrent prostate cancer: evaluation in 1007 patients. Eur J Nucl Med Mol Imaging. 2017;44:1258-1268.

23. Afshar-Oromieh A, Haberkorn U, Hadaschik B, et al. PET/MRI with a ${ }^{68} \mathrm{Ga}-$ PSMA ligand for the detection of prostate cancer. Eur J Nucl Med Mol Imaging. 2013;40:1629-1630.

24. Afshar-Oromieh A, Zechmann CM, Malcher A, et al. Comparison of PET imaging with a ${ }^{68} \mathrm{Ga}$-labelled PSMA ligand and ${ }^{18} \mathrm{~F}$-choline-based PET/CT for the diagnosis of recurrent prostate cancer. Eur J Nucl Med Mol Imaging. 2014;41:11-20.

25. Perera M, Papa N, Christidis D, et al. Sensitivity, specificity, and predictors of positive ${ }^{68} \mathrm{Ga}$-prostate-specific membrane antigen positron emission tomography in advanced prostate cancer: a systematic review and meta-analysis. Eur Urol. 2016;70:926-937.

26. Rowe SP, Gage KL, Faraj SF, et al. ${ }^{18}$ F-DCFBC PET/CT for PSMA-based detection and characterization of primary prostate cancer. J Nucl Med. 2015;56:1003-1010.

27. Cho SY, Gage KL, Mease RC, et al. Biodistribution, tumor detection, and radiation dosimetry of ${ }^{18} \mathrm{~F}$-DCFBC, a low-molecular-weight inhibitor of prostatespecific membrane antigen, in patients with metastatic prostate cancer. $\mathrm{J} \mathrm{Nucl}$ Med. 2012;53:1883-1891.

28. Rowe SP, Macura KJ, Ciarallo A, et al. Comparison of prostate-specific membrane antigen-based ${ }^{18} \mathrm{~F}-\mathrm{DCFBC}$ PET/CT to conventional imaging modalities for detection of hormone-naïve and castration-resistant metastatic prostate cancer. J Nucl Med. 2016;57:46-53.

29. Szabo Z, Mena E, Rowe SP, et al. Initial evaluation of $\left[{ }^{18} \mathrm{~F}\right] \mathrm{DCFPyL}$ for prostatespecific membrane antigen (PSMA)-targeted PET imaging of prostate cancer. Mol Imaging Biol. 2015;17:565-574.

30. Rowe SP, Macura KJ, Mena E, et al. PSMA-based [ $\left[{ }^{18} \mathrm{~F}\right] \mathrm{DCFPyL}$ PET/CT is superior to conventional imaging for lesion detection in patients with metastatic prostate cancer. Mol Imaging Biol. 2016;18:411-419.

31. Giesel FL, Kesch C, Yun M, et al. ${ }^{18}$ F-PSMA-1007 PET/CT detects micrometastases in a patient with biochemically recurrent prostate cancer. Clin Genitourin Cancer. 2017;15:e497-e499.

32. Budäus L, Leyh-Bannurah S-R, Salomon G, et al. Initial experience of ${ }^{68} \mathrm{Ga}-$ PSMA PET/Ct imaging in high-risk prostate cancer patients prior to radical prostatectomy. Eur Urol. 2016;69:393-396. 
33. Herlemann A, Wenter V, Kretschmer A, et al. ${ }^{68}$ Ga-PSMA positron emission tomography/computed tomography provides accurate staging of lymph node regions prior to lymph node dissection in patients with prostate cancer. Eur Urol. 2016;70:553-557.

34. Maurer T, Gschwend JE, Rauscher I, et al. Diagnostic efficacy of ${ }^{68}$ galliumPSMA positron emission tomography compared to conventional imaging for lymph node staging of 130 consecutive patients with intermediate to high risk prostate cancer. J Urol. 2016;195:1436-1443.

35. Sahlmann C-O, Meller B, Bouter C, et al. Biphasic ${ }^{68}$ Ga-PSMA-HBED-CC$\mathrm{PET} / \mathrm{CT}$ in patients with recurrent and high-risk prostate carcinoma. Eur J Nucl Med Mol Imaging. 2016;43:898-905.

36. Thompson IM, Tangen CM, Paradelo J, et al. Adjuvant radiotherapy for pathologic T3NOM0 prostate cancer significantly reduces risk of metastases and improves survival: long-term followup of a randomized clinical trial. J Urol. 2009;181:956-962.

37. Bluemel C, Krebs M, Polat B, et al. ${ }^{68} \mathrm{Ga}$-PSMA-PET/CT in patients with biochemical prostate cancer recurrence and negative ${ }^{18} \mathrm{~F}$-choline-PET/CT. Clin $\mathrm{Nucl}$ Med. 2016;41:515-521.

38. Pandit-Taskar N, O'Donoghue JA, Beylergil V, et al. ${ }^{89} \mathrm{Zr}$-huJ591 immuno-PET imaging in patients with advanced metastatic prostate cancer. Eur J Nucl Med Mol Imaging. 2014;41:2093-2105.

39. Barrett JA, Coleman RE, Goldsmith SJ, et al. First-in-man evaluation of 2 highaffinity PSMA-avid small molecules for imaging prostate cancer. $\mathrm{J} \mathrm{Nucl} \mathrm{Med}$. 2013;54:380-387.

40. Afshar-Oromieh A, Malcher A, Eder M, et al. PET imaging with a $\left[{ }^{68} \mathrm{Ga}\right]$ galliumlabelled PSMA ligand for the diagnosis of prostate cancer: biodistribution in humans and first evaluation of tumour lesions. Eur J Nucl Med Mol Imaging. 2013;40:486-495.

41. Afshar-Oromieh A, Hetzheim H, Kratochwil C, et al. The theranostic PSMA ligand PSMA-617 in the diagnosis of prostate cancer by PET/CT: biodistribution in humans, radiation dosimetry, and first evaluation of tumor lesions. J Nucl Med. 2015;56:1697-1705.

42. Ahmadzadehfar H, Rahbar K, Kürpig S, et al. Early side effects and first results of radioligand therapy with ${ }^{177} \mathrm{Lu}-\mathrm{DKFZ}-617$ PSMA of castrateresistant metastatic prostate cancer: a two-centre study. EJNMMI Res. 2015;5: 114.

43. Kratochwil C, Giesel FL, Stefanova M, et al. PSMA-targeted radionuclide therapy of metastatic castration-resistant prostate cancer with ${ }^{177} \mathrm{Lu}$-labeled PSMA617. J Nucl Med. 2016;57:1170-1176.

44. Bräuer A, Grubert LS, Roll W, et al. ${ }^{177}$ Lu-PSMA-617 radioligand therapy and outcome in patients with metastasized castration-resistant prostate cancer. Eur $J$ Nucl Med Mol Imaging. 2017;44:1663-1670.

45. Ahmadzadehfar H, Wegen S, Yordanova A, et al. Overall survival and response pattern of castration-resistant metastatic prostate cancer to multiple cycles of radioligand therapy using $\left[{ }^{177} \mathrm{Lu}\right] \mathrm{Lu}-\mathrm{PSMA}-617$. Eur J Nucl Med Mol Imaging. 2017;44:1448-1454.

46. Fendler WP, Reinhardt S, Ilhan H, et al. Preliminary experience with dosimetry, response and patient reported outcome after ${ }^{177}$ Lu-PSMA-617 therapy for metastatic castration-resistant prostate cancer. Oncotarget. 2017;8:35813590 .

47. Rahbar K, Ahmadzadehfar H, Kratochwil C, et al. German multicenter study investigating ${ }^{177} \mathrm{Lu}-\mathrm{PSMA}-617$ radioligand therapy in advanced prostate cancer patients. J Nucl Med. 2017;58:85-90.

48. Baum RP, Kulkarni HR, Schuchardt C, et al. ${ }^{177} \mathrm{Lu}$-labeled prostate-specific membrane antigen radioligand therapy of metastatic castration-resistant prostate cancer: safety and efficacy. J Nucl Med. 2016;57:1006-1013.

49. Kratochwil C, Bruchertseifer F, Rathke H, et al. Targeted $\alpha$-therapy of metastatic castration-resistant prostate cancer with ${ }^{225}$ Ac-PSMA-617: dosimetry estimate and empiric dose finding. J Nucl Med. 2017;58:1624-1631. 